\title{
Eine neue Strategie "eHealth» für die Schweiz
}

\section{Reinhold Sojer}

Dr., Wissenschaftlicher Mitarbeiter, Stv. Abteilungsleiter Digitalisierung eHealth, FMH
Die neue Strategie eHealth Schweiz ist da. Bund und Kantone haben in einem Entwurf, welcher sich bis zum 10. Dezember 2017 in Anhörung befand [1], die «Strategie eHealth Schweiz» aus dem Jahr 2007 fortgeführt. Die Strategie umfasst drei Handlungsfelder mit 27 Zielen, die in Phasen im Zeitraum von 2018 bis 2022 umgesetzt werden sollen. Mit den festgelegten Handlungsfeldern sollen die Digitalisierung gefördert, koordiniert und sowohl Gesundheitsfachpersonen als auch Patientinnen und Patienten zur Digitalisierung befähigt werden [2].

Die Digitalisierung als Treiber eines tiefergreifenden Strukturwandels hat Auswirkungen auf fast alle gesellschaftlichen und wirtschaftlichen Bereiche. Mit Digitalisierung wird oftmals eine Technologisierung, die Adaption von neuen Informationstechnologien verstanden, welche zunehmend analoge Hilfsmittel verdrängen. Dieser Prozess hat in den letzten Jahrzehnten durch die Verfügbarkeit leistungsfähiger Rechensysteme und

\section{Die Strategie umfasst drei Handlungsfelder mit 27 Zielen, die in Phasen im Zeitraum von 2018 bis 2022 umgesetzt werden sollen.}

einer moderaten Kostenentwicklung rasant Fahrt aufgenommen. Intel-Gründer Gordon Moore prophezeite 1965, dass sich die Anzahl der integrierten Schaltkreise auf Computerchips alle 12 Monate verdoppeln werden, und in einem Erratum korrigierte Moore 10 Jahre später die exponentielle Zunahme auf 24 Monate [3]. Diese Aussage hat mehrere Jahrzehnte die Entwicklung von leistungsfähigen Computern im Sinne einer selbsterfüllenden Prophezeiung beherrscht. Heute gilt sie jedoch nicht mehr, und ein Grund waren die Entwicklung von Cloud-Computing (siehe [4]) und die Vernetzung von Smart-Devices, hochspezialisierter Geräte, ausgestattet mit Sensoren, welche autonom oder interaktiv in einer vernetzten Umgebung agieren [5]. Beispielhaft für diese Veränderung im Gesundheitswesen steht die Entwicklung der Anwendung «Streams» im Royal Free, London. «Streams» ist ein vollständig digitaler klinischer Behandlungspfad für die Erkennung eines akuten Nierenversagens, basierend auf vernetzten, mobilen Technologien [6].
Jede Revolution hat ihre Opfer, so auch die digitale Revolution. Einerseits treten mit der Technologisierung neue Themen wie Datenschutz, ${ }^{1}$ Risiken der digitalen Vernetzung und Transparenz oder die digitale Kompetenz in den Vordergrund. Andererseits sind Prozesse, die sehr stark abhängig sind von physischen Einrichtungen und Personal, disruptiven Veränderungen unterworfen. Wir bewegen uns hiermit zum Kern einer Strategie für die Digitalisierung im Gesundheitswesen: Die digitale Transformation und nicht die Digitalisierung (Technologisierung) lässt neue Prozesse und Dienstleistungen entstehen, mit denen Aufgaben und Probleme effizienter und wirksamer gelöst werden können. Wie weit weg der Strategieentwurf von Bund und Kantonen von der Vorstellung einer Prozesstransformation ist, zeigt die Zielformulierung «Ersatz von papierbasierten durch digitale Prozesse». Auch findet die Transformation zunächst auf der gesellschaftlichen und meist erst später auf der geschäftlichen Ebene statt. Die Transformation auf geschäftlicher Ebene orientiert sich hierbei an den Bedürfnissen der Anwender oder Konsumenten der neuen Technologien und nicht umgekehrt. Die Koordination der Digitalisierung, wie in der Strategie als Handlungsfeld formuliert, kann daher keine Aufgabe des Staates sein. Seine Aufgabe muss es sein, Innovationen zu fördern und wo notwendig Anreize zu setzen. Was bleibt, ist die Schaffung positiver Rahmenbedingungen, aus gesellschaftlicher Sicht die Positionierung des Menschen in das Zentrum der Strategie sowie die Motivation der professionellen Anwender.

\section{Die digitale Transformation lässt neue Prozesse} und Dienstleistungen entstehen, mit denen Aufgaben und Probleme effizienter und wirksamer gelöst werden können.

Auch wenn in der Strategie eHealth Schweiz 1.0 festgehalten wurde, dass der Mensch und damit die Patientinnen und Patienten im Mittelpunkt einer eHealthStrategie stehen müssen, war die Umsetzung sehr technisch geprägt. Die Strategie eHealth Schweiz 2.0 steht im Zeichen der Einführung und Verbreitung EPD, 


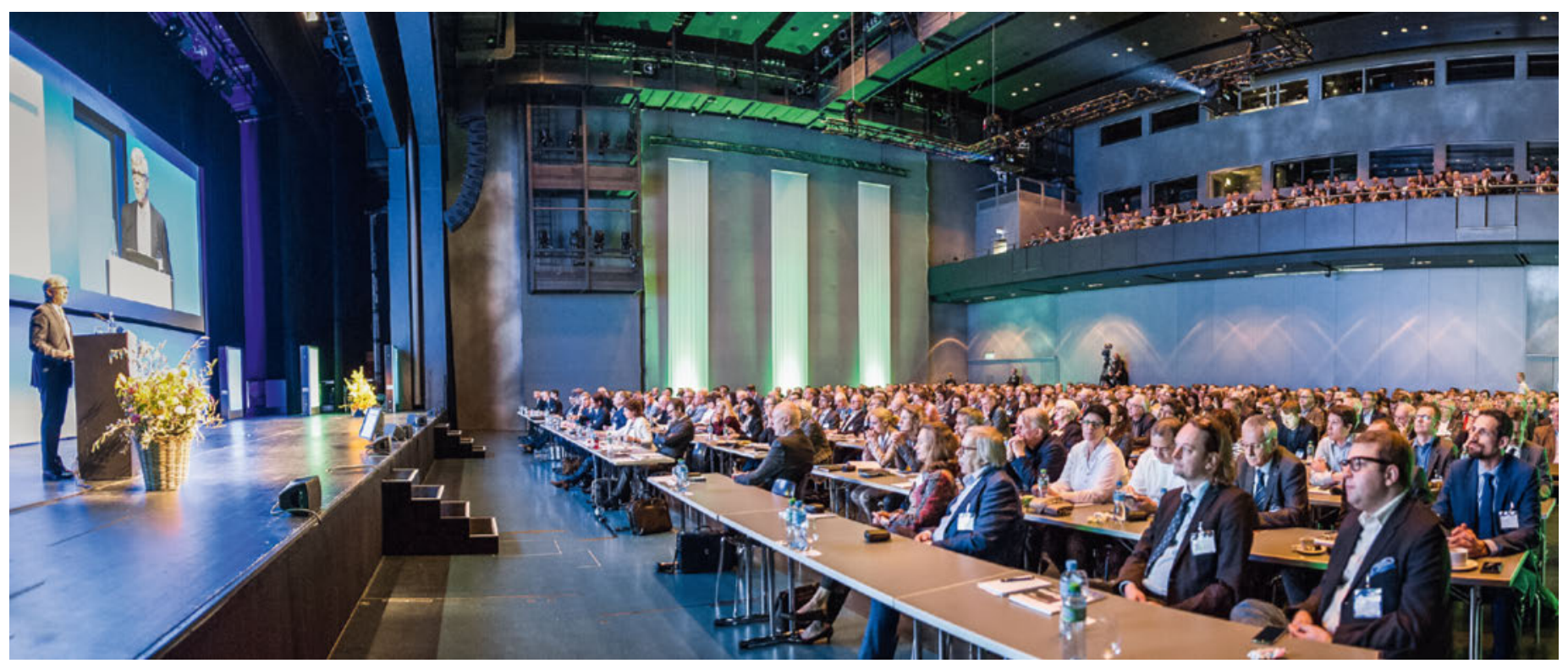

\section{BE SMART - Medizin mit Augenmass}

BE SMART - die Suche nach klugen Entscheidungen für eine Medizin mit Augenmass steht im Mittelpunkt derTrendtage Gesundheit 2018. Im Zuge der aktuellen Diskussionen, die nicht nur in der Politik, sondern vermehrt auch in der Öffentlichkeit geführt werden, wollen die Trendtage Gesundheit einen aktiven Beitrag zu einem konstruktiven Dialog leisten. Wir wollen uns insbesondere folgenden Fragen widmen: Wo und warum gibt es Fehlversorgung (Overuse, Underuse), wie sind die regionalen und internationalen Unterschiede? Was könnte man dagegen tun? Braucht es mehr (verbindliche) Guidelines oder sind staatliche Vorgaben nötig? Wie müssten diese ausgestaltet sein? Welche Behandlungen will man sich zu welchem Preis und Nutzen leisten, und warum kann weniger auch mal mehr sein? Welches sind die Rollen der Leistungserbringer, der Politik oder der Versicherer? Welche ethischen Anliegen sind zu beachten, und wie könnte die Digitalisierung die Prozesse zusätzlich optimieren? Die zentrale Frage ist dabei immer, was letztlich für die Patientinnen und Patienten die kluge Therapie ist.

www.trendtage-gesundheit.ch/de/ueber_uns/Trendtage_Gesundheit

ergänzt durch weiteren regulatorischen Handlungsbedarf zu Themen wie Mobile Health. Dort, wo ansatzweise der Mensch in den Mittelpunkt rückt - die Befä-

\section{Neben der Befähigung und Motivation der Patienten bedarf es einer Motivation derjeni- gen, welche die Daten ohne weitere finanziellen Anreize in das System EPD einspeisen.}

\begin{tabular}{l}
\hline Korrespondenz: \\
Dr. rer. biol. hum. \\
Reinhold Sojer \\
Wissenschaftlicher Mitar- \\
beiter / Stv. Leiter Abteilung \\
Digitalisierung / eHealth- \\
FMH Verbindung der \\
Schweizer Ärztinnen und \\
Ärzte \\
Elfenstrasse 18 \\
Postfach 300 \\
CH-3000 Bern 15 \\
Tel. 031 3591204 \\
reinhold.sojer[at]fmh.ch
\end{tabular}

higung von Patientinnen und Patienten zur digitalen Gesundheitskompetenz (eHealth Literacy) -, müssen nun konkrete Massnahmen benannt werden. Dies ist eine umfassende Aufgabe und darf sich nicht nur, wie im Strategieentwurf beschrieben, auf die Modifikation von Projekten zur Gesundheitskompetenz beschränken. Neben der Befähigung und Motivation der Patientinnen und Patienten bedarf es einer Motivation derjenigen, welche die Daten ohne weitere finanziellen Anreize in das System EPD einspeisen. Nicht nur die technischen Schnittstellen müssen nahtlos sein, sondern auch die Prozessanbindung an die Primärdokumentation.

Vor etwa 10 Jahren hat Martin Denz in der Schweizerischen Ärztezeitung die Konsequenzen einer nationalen eHealth-Strategie aufgezeigt, und immer noch gilt, dass eHealth ein Ausdruck eines anstehenden Transformationsprozesses ist, bei dem durch den Einsatz von ICT neue Prozesse und Dienstleistungen entstehen, und auch, dass Strukturen und Prozesse bedeutungslos werden, die allzu lange weder auf ihren Sinn noch ihre Bedarfs- und Nutzenorientierung hinterfragt worden sind [7].

\section{Bildnachweis}

Trendtage Gesundheit Luzern 2017

\section{Literatur}

1 Koordinationsorgan eHealth Bund-Kantone, Strategie eHealth Schweiz 2.0 2018-2022 (Entwurf vom 5. September 2017), 2017. [Online]. Available: https://www.e-health-suisse.ch/fileadmin/ user_upload/Dokumente/2017/D/170911_Entwurf_Strategie_ eHealth 2.0 d.pdf. [Accessed: 10-Dec-2017].

2 Bundesamt für Gesundheit, Strategie 'eHealth' Schweiz, 2007. [Online]. Available: https://www.e-health-suisse.ch/fileadmin/ user_upload/Dokumente/2007_2008/D/070627_Strategie_ eHealth_Schweiz_D.pdf. [Accessed: 10-Dec-2017].

3 Moore GE. Cramming more components onto integrated circuits (Reprinted from Electronics, p. 114-7, April 19, 1965). Proc Ieee. 1965;86(1):82-5.

4 Kessler T. Informationssicherheit beim Cloud Computing. Schweizerische Ärztezeitung. 2017;98(45):1493-4.

5 Waldrop MM. The chips are down for Moore's law. Nature. 2016;530(7589):144-7.

6 Connell A, Montgomery H, Morris S, Nightingale C, Stanley S, Emerson M, et al. Service evaluation of the implementation of a digitally-enabled care pathway for the recognition and management of acute kidney injury. F1000Research. 2017;6:1033.

7 Denz M. Konsequenzen der nationalen E-Health-Strategie. Schweizerische Ärztezeitung. 2017;88(9):378-82. 